\title{
MRI Diagnosis and Pathological Analysis of Intracranial Tumors
}

\author{
Peng Fang ${ }^{1,2}$ \\ ${ }^{1}$ Department of Radiology, Henan Province Hospital of Traditional Chinese Medicine, Zhengzhou, Henan, \\ China \\ ${ }^{2}$ Hospital of Henan University of Traditional Chinese Medicine, Zhengzhou, Henan 450002, China
}

Keywords: Intracranial tumor, Magnetic resonance imaging, MRI, Pathological comparison

\begin{abstract}
At present, the diagnosis of intracranial tumors is mainly through CT or MRI, and surgery is mainly used for the treatment of intracranial tumors. Therefore, preoperative diagnosis is very important, and the location of the lesion should be clarified, which is conducive to the formulation of surgical plan. Intracranial tumor is a common tumor, accounting for about $5 \%$ of human tumors. The clinical manifestations of patients are lack of specificity, so diagnosis and differential diagnosis are needed. The application of imaging examination in intracranial tumors has important clinical application value in the diagnosis and differential diagnosis. This paper starts with the diagnosis and pathological analysis of intracranial tumors.
\end{abstract}

\section{Introduction}

The MRI diagnosis of intracranial tumors is significantly better than other imaging diagnosis, which is the key basis for the diagnosis of intracranial tumors. From the clinical observation of MRI diagnosis at home and abroad, it is of great significance for the early detection of intracranial tumors, and for the middle and late stages. The ward judgment and surgical treatment of intracranial tumors have important value.

\section{Overview of Intracranial Tumor}

Primary intracranial tumor is a kind of primary tumor. It is frequently occurred in brain. The incidence rate of intracranial tumors has been increasing in recent years. According to statistics, intracranial tumors account for about $5 \%$ of the whole-body tumors $70 \%$ of children's tumors, 20 to $30 \%$ other malignant tumors will eventually be transferred into the intracranial part. Due to the expanding and infiltrating growth of tumor, once it occupies a certain space in the brain, regardless of its nature is benign or malignant, it will have an impact on the brain. Patients with this disease are lack of specific performance, and most of them are in the late stage when they are admitted to hospital, which delays the best treatment time [1]. It is important to choose a reliable diagnosis method as soon as possible. Magnetic resonance imaging (MRI) is an important means of early and accurate diagnosis of intracranial tumors, which plays an important role in clinical diagnosis. In order to further explore the value of MRI in the diagnosis of PIL, it is necessary to make a comprehensive diagnosis based on clinical observation that patients have different degrees of dizziness, severe headache, memory loss, vision decline, unclear speech, unilateral limb weakness and other symptoms.

\section{MRI Diagnosis of Intracranial Tumors}

\subsection{Inclusive Criteria and Exclusion Criteria}

Inclusive criteria: (1) PIL was confirmed by clinical and postoperative pathology [2]; (2) age: 25-65 years; (3) The data were complete and examined by MRI. Exclusion criteria: (1) poor willingness to cooperate and disobey the arrangement; (2) poor compliance; (3) other types of unknown intracranial diseases or tumors in other parts; (4) disagreement with the research method. The research needs to be approved by relevant authorities and medical ethics committee. 


\subsection{Inspection Method}

MRI examination method: MRI scanning parameters were $6-8 \mathrm{~mm}$ slice thickness, $0.4-0.6 \mathrm{~mm}$ interval, 256×256 matrix. After injection of $0.1 \mathrm{ml} / \mathrm{kg}$ meglumine gadopentetate, three-dimensional images were obtained by head transverse, coronal and sagittal enhanced scanning.

\subsection{Observation Index}

Two senior doctors in the imaging department of the hospital used the double-blind method for diagnosis and differentiation, and the same diagnosis result is the final diagnosis result. If the diagnosis results of the two were inconsistent, it should be decided by the doctor with senior title or above. The detection rate and accuracy of MRI in the diagnosis and differential diagnosis of intracranial tumors were evaluated according to the pathological results.

\section{Analysis of MRI Diagnosis of Intracranial Tumors}

\subsection{Diagnostic Criteria}

The incidence rate of intracranial tumors is increasing year by year, which is more harmful and needs more attention. Therefore, early MRI diagnosis is very important. The diagnosis and treatment of intracranial tumors should be made as soon as possible. Imaging technology is an effective method for preoperative diagnosis of intracranial tumors. Combined with literature review, the diagnostic criteria of the disease are: (1) intracranial or intraspinal lesions as the main symptoms; (2) pathological confirmation; (3) tumors at other sites; (4) no follow-up of all parts of the body, blood and bone marrow examination show pathological changes [3].

\subsection{MRI Diagnosis as a Reliable Imaging Technique}

In recent years, MRI has become an effective method for the diagnosis of PIL. From the application effect of many years, MRI examination is the main method of diagnosis for tumor patients. From the perspective of imaging technology, for patients with intracranial tumors, including left temporal lobe, left frontal lobe, right cerebellum, corpus callosum, MRI diagnosis signal characteristics mostly demonstrate isointense in T1WI, less in low signal and mixed signal, high signal in T2WI, mixed signal in the rest, and cystic change, necrosis and hemorrhage are rare. After enhanced scanning, homogeneous enhancement appears with heterogeneous enhancement and other peritumoral edema of different degrees. The ADC value of the tumor was not shown by DWI sequence scanning, which can better reflect the characteristics of limited diffusion of PIL. DWI is helpful in the differential diagnosis of intracranial PIL. It can be seen that MRI has its own imaging characteristics in the diagnosis of PIL, which provides a basis for the diagnosis of PIL. In order to improve the clinical diagnosis effect, differential diagnosis should be done well, such as glioma, which will not only involve corpus callosum, irregular shape, lymphoma, advanced glioma, cystic change, hemorrhage, necrosis, etc., but also lead to uneven MRI signal and obvious peritumoral edema [4]. For example, metastasis, PIL and metastatic tumor are sometimes difficult to differentiate. Most of the metastases have history, and the primary focus is basically clear. The signals of lesions are mainly inhomogeneous with necrosis and liquefaction. Contrast enhanced scan showed round enhancement, different from some tumors. MRI diagnosis of PIL has high diagnostic value, and it can show some unique MRI features. Through the analysis of its performance, we can accurately determine most of the intracranial PIL, and can locate the intracranial tumor lesions and other intracranial tumors, which provides a certain reference for surgical treatment.

\section{Pathological Analysis and Differentiation}

\subsection{Postoperative Pathology and Immunohistochemistry}

(1) Naked eye observation: tumor without capsule, grayish-yellow, grayish-white, whether there is visible cystic cavity, mostly yellow and light yellow liquid, such as a small amount of bloody 
fluid; (2) Observation under microscope: the tumor is composed of dense two-phase slender tumor cells, one end is filiform with vascular proliferation reaction. Dense tumor cells, loose Rosenthal fibers and eosinophilic bodies can be seen in the connective tissue of the lesion. Immunohistochemistry shows glial fibrin (GFAP) positive.

\subsection{Symptoms of Intracranial Tumors}

General surgical resection of intracranial tumors has good therapeutic effect. Adult cerebral hemispheric intracranial tumors may recur. Intracranial tumors are common in cerebellar hemisphere, sellar region, brainstem and pineal region. The clinical specificity of sellar region of intracranial tumors is not obvious, which is related to the location of lesions, the size of tumors and the degree of hydrocephalus. Intracranial hypertension is the main manifestation of cerebellar tumors, accompanied by walking instability, blurred vision and ataxia. The main clinical symptoms of sellar tumors are headache, vomiting, papilledema and visual impairment. The first symptoms of cerebral hemisphere tumors include headache, convulsion, coma, fecal incontinence, etc. Visual acuity and visual field defect in optic nerve area are accompanied by optic disc pallor, optic nerve atrophy and exophthalmos. Headache and dizziness are the first symptoms of the patient.

\subsection{Pathological Feature of Intracranial Tumors}

The pathological feature of intracranial tumors is actually a mass formed by normal differentiation of astrocytes. In typical intracranial tumors, dense tumor cells and loose connective tissue crisscross with each other, and cystic tissues of different sizes and vascular proliferation can be observed. The cystic changes of intracranial tumors are not liquefaction necrosis caused by ischemia, but the matrix secreted by astrocytes in proliferative state. From the pathological changes, cystic inversion is a sign of good differentiation of cancer cells and one of the characteristics of benign tumors. It is reported that the eosinophilic bodies under microscope show low grade, slow growth and good prognosis.

\subsection{Imaging Findings}

\subsubsection{Typical Imaging Findings of Intracranial Tumors}

There are two imaging manifestations: one is cystic nodule with wall, which is obviously enhanced after contrast-enhanced while the cystic wall is not enhanced. The other is solid mass (solid part accounts for 60\%), and the solid part is obviously enhanced on enhanced scanning. DWI shows that cystic components of intracranial tumor are hypointense on DWI, and ADC value of solid component is high, indicating that solid component of PA was sparse. MRS shows that the peaks of NAA and Cr decrease, while the peaks of CHO and Lac increase slightly. For example, cystic mural nodular tumors located in the cerebellum are significantly enhanced after enhancement, which is consistent with the literature reports. For some grade I gliomas, peritumoral edema is rare and mild, which is an important feature of diagnosis. Some studies suggest that the substantial part of intracranial tumor is obviously enhanced, which can not be considered as the damage of blood-brain barrier of malignant tumor. It may be related to the abundance of tumor blood vessels, which is porous capillaries. In addition, in nodular intracranial tumors with cystic wall, the enhanced wall of the cyst does not represent the presence of tumor cells caused by vascular proliferation.

\subsubsection{Atypical Imaging Findings}

Atypical imaging findings include cystic solid lesions (solid part accounts for less than 60\%), simple cystic lesions, intratumoral calcification, hemorrhage, atypical enhancement mode, leptomeningeal dissemination, etc. There are necrotic cystic changes in the central area of cystic and solid intracranial tumors. The cystic part accounts for $60 \%$, and the solid part is enhanced by enhanced scanning. Simple cystic disease is rare, and cystic wall enhancement is not enhanced. This group of cases was rare, no simple cystic lesions. Intracerebral hemorrhage is usually rare. The hemorrhage in the tumor is thought to be caused by endothelial cell proliferation and vascular wall 
fiber structure. For example, the cystic and solid lesions in pons area show mixed signal, less than $50 \%$ of solid part is bleeding and enhanced scanning shows circular enhancement. DWI mainly shows low signal. Preoperative bleeding was misdiagnosed as angiogenic tumor. Thus, familiarity with atypical imaging findings can reduce the misdiagnosis rate. There was no calcification in the intracranial tumors because of the small number of cases. Generally, the calcification rate is $15 \%$ [4], which is mainly caused by degenerative changes such as tumor necrosis, bleeding and lymphocyte infiltration. Atypical enhancement includes heterogeneous patchy enhancement, nodular enhancement, round enhancement and mild enhancement, which is easy to be misdiagnosed. Some studies have shown that the proportion of loose and dense areas in tumor is different, which leads to different ways of tumor enhancement.

\section{Differential Diagnosis}

\subsection{Hemangioblastoma Can Be Divided into Round and Quasi Round Shape.}

Contrast-enhanced MRI can show mural nodules, which are more obvious than pilocytic astrocytoma, forming the so-called "wall lamp" sign. Hemangioblastoma mainly occurs in adult women and originates around the midline of cerebellum. Blood vessels can be seen near the tumor wall to distinguish the tumor. The stromal cells of hemangioblastoma nodule contain fat. MRS show high lip peak, but NAA peak and Lac peak are not found.

\subsection{Medulloblastoma is an Infratentorial Malignant Tumor in Children.}

Medulloblastoma usually occurrs in children under 15 years old. The ratio of male to female was 3.8:1. The tumor is highly malignant and grows rapidly. The tumor will grow into the fourth ventricle and is solid. Medulloblastoma has a high degree of malignancy, and its blood-brain barrier is damaged in varying degrees. PWI can reflect the tissue vascular proliferation and blood perfusion, which is helpful to differentiate medulloblastoma.

\subsection{Craniopharyngioma is Common in Children and Can Occur in Adults.}

Cystic and cystic solid craniopharyngiomas have cystic change, calcification, hemorrhage and cholesterol crystals. MRI has characteristic high and low mixed-signal, and PA calcification is rare; solid craniopharyngioma is rare, accompanied by pituitary and hypothalamic endocrine symptoms.

\subsection{Mucinous Pilocytic Astrocytoma}

Myxoid astrocytoma (PMA) is aggressive and has a high recurrence rate. The incidence of PMA in infants is higher than that in PA. There are more solid components and less cystic components in PMA. The CHO / Cr ratio of PMA lesions is similar to that of PA, but slightly higher than that of $\mathrm{PA}$, indicating strong infiltration.

\section{Conclusion}

Intracranial tumors have the following characteristics: (1) slow growth; (2) low differentiation; (3) good prognosis. In MRI examination, most of the patients showed typical manifestations, including cystic wall nodular type and solid mass. The parenchymal part was significantly enhanced, and peritumoral edema was mild and absent. Some patients showed atypical manifestations. Familiarity with atypical manifestations can reduce the misdiagnosis rate of the disease. In clinical work, if the imaging can be effectively combined with pathology, it will not only contribute to the formulation of clinical surgery and treatment plan, but also help to reduce postoperative complications and improve the quality of life of patients.

\section{References}

[1] Wang Haili, Zhang Zhongmian. Clinical Manifestations, Imaging Features and Pathological Diagnosis of Primary Central Nervous System Lymphoma. Journal of Experimental Hematology, 
vol. 26, no.1, pp.171-176, 2018.

[2] Yu Hui, Yu Yongcheng, Liu Jing, et al. Clinical and MRI findings of brain lymphoma. Chinese Journal of Radiology, vol.52, no.1, pp.58-60, 2018.

[3] Zhang Huan. Clinical characteristics of 23 cases of primary central nervous system lymphoma. Shandong Medical Journal, vol.56, no.44, pp. 81-83, 2016.

[4] Zhang Xiuling, Shi Changhong. Value of CT and MRI in diagnosis of intracranial primary central nervous system lymphoma. Chinese Imaging Journal of Integrated Traditional and Western Medicine, vol.16, no.1, pp.15-17, 25, 2018. 